\title{
Extending the SRIV Identification Algorithm to MIMO LMFD Models
}

\author{
Mohamed Akroum ${ }^{\dagger}$ and Kamel Hariche*
}

\begin{abstract}
In this paper the Simplified Refined Instrumental Variable (SRIV) identification algorithm for SISO systems is extended to MIMO systems described by a Left Matrix Fraction Description (LMFD). The performance of the extended algorithm is compared to the well-known MIMO four-step instrumental variable (IV4) algorithm. Monte Carlo simulations for different signal to noise ratios are conducted to assess the performance of the algorithm. Moreover, the algorithm is applied to a simulated quadruple tank process.
\end{abstract}

Keywords: MIMO system identification, SRIV, LMFD, IV4, Steiglitz-McBride

\section{Introduction}

Many different identification methods have been proposed for both SISO and MIMO systems. Among these we can mention the PEM and n4sid [1] for the identification of state space models, and the ARX, IVX and IV4 methods [1] for systems modeled by a Left Matrix Fraction Description. These methods have been implemented and are available in the Matlab System identification toolbox [2].

An interesting identification algorithm was proposed by Young [3] [4] and is referred to as the Simplified Refined Instrumental Variable (SRIV). It is an optimal instrumental variable algorithm proposed for the identification of noisy SISO systems.

It is the purpose of this paper to extend the algorithm for the identification of noisy MIMO systems described by a Left Matrix Fraction Description. The performance of the extended algorithm is then compared to that of the MIMO IV4 [1], [2] algorithm used as a benchmark.

In this paper the m-input $\mathrm{p}$-output noisy multivariable system is assumed to be modeled in matrix fraction description form as:

where

$$
y[k]=A^{-1}\left(q^{-1}\right) B\left(q^{-1}\right) u[k]+e[k]
$$

$$
\begin{aligned}
& A\left(q^{-1}\right)=I_{p}+A_{1} q^{-1}+\ldots A_{n a} q^{-n a} \\
& B\left(q^{-1}\right)=B_{1} q^{-1}+\ldots B_{n b} q^{-n b}
\end{aligned}
$$

$e[k]$ is a white noise vector and $\mathrm{q}$ is the shift operator.

$\dagger$ Corresponding Author: Dept. of Electrical and Electronic Engineering, M'hamed Bougara University, Boumerdes, Algeria (m_akroum@yahoo.com)

* Dept. of Electrical and Electronic Engineering, M'hamed Bougara University, Boumerdes, Algeria (khariche@yahoo.com) Received 3 March, 2008 ; Accepted 13 November, 2008

\section{The MIMO IV4 Algorithm}

Given a MIMO system modeled as:

$$
y[k]=A^{-1}\left(q^{-1}\right) B\left(q^{-1}\right) u[k]+e[k]
$$

The objective is to identify the matrix coefficients $A_{i} \in R^{p \times p}$ and $B_{i} \in R^{p \times m}$ of the matrix polynomials $A\left(q^{-1}\right)$ and $B\left(q^{-1}\right)$.

Defining a new vector $v[k]=A\left(q^{-1}\right) e[k]$ we can write:

$$
\begin{gathered}
v^{T}[k]=y^{T}[k]+y^{T}[k-1] A_{1}^{T} \ldots+y^{T}[k-n a] A_{n a}^{T} \\
-u^{T}[k] B_{0}^{T} \ldots-u^{T}[k-n b] B_{n b}^{T}
\end{gathered}
$$

or,

$$
v^{T}[k]=y^{T}[k]-\varphi^{T}[k] \theta
$$

where

$$
\begin{aligned}
& \varphi^{T}[k]=\left[-y^{T}[k-1] \ldots-y^{T}[k-n a] \quad u^{T}[k-1] \ldots u^{T}[k-n b]\right] \\
& \text { and } \theta=\left[\begin{array}{llllllll}
A_{1}^{T} & . & . & A_{n a}^{T} & B_{1}^{T} & . & . & B_{n b}^{T}
\end{array}\right]^{T}
\end{aligned}
$$

The MIMO IV4 algorithm [1], [2] may be summarized as follows:

\section{Algorithm (IV4 Algorithm)}

1- Determine the Least Squares estimate $\hat{\theta}_{l s}$ using i/o data as:

$$
\hat{\theta}_{l s}=\left[\Phi^{T} \Phi\right]^{-1} \Phi^{T} Y
$$

where 
$Y=\left[\begin{array}{c}y^{T}[n+1,:] \\ \cdot \\ \cdot \\ y^{T}[N,:]\end{array}\right]$

$\Phi=\left[\Phi_{y} \vdots \Phi_{u}\right]$

$\left.\Phi_{y}=\left[\begin{array}{cc|c|c}-y^{T}[n,:] & \ldots & -y^{T}\left[n-n_{a}+1,:\right] \\ \cdot & \ldots & \cdot \\ \cdot & \cdots & \cdot \\ \cdot & \cdots & \cdot \\ -y^{T}[N-1,:] & \cdots & -y^{T}\left[N-n_{a},:\right]\end{array}\right]\right]$

$\left.\Phi_{u}=\left[\begin{array}{cc|c|c}u^{T}[n,:] & \ldots & u^{T}\left[n-n_{b}+1,:\right] \\ \cdot & \ldots & \cdot \\ \cdot & \ldots & \cdot \\ \cdot & \ldots & \cdot \\ u^{T}[N-1,:] & \cdots & u^{T}\left[N-n_{b},:\right]\end{array}\right]\right]$

and $n=n a$

2- Simulate the model output

$z[k]=A^{-1}\left(q^{-1}\right) B\left(q^{-1}\right) u[k]$

to get $z[k]$

3- Estimate the parameters $\hat{\theta}_{i v}$ as:

$$
\hat{\theta_{i v}}=\left[\Psi^{T} \Phi\right]^{-1} \Psi^{T} Y
$$

where

$$
\Psi=\left[\Phi_{z} \vdots \Phi_{u}\right]
$$

$\left.\Phi_{z}=\left[\begin{array}{cc|c|c}-z^{T}[n,:] & \ldots & -z^{T}\left[n-n_{a}+1,:\right] \\ \cdot & \ldots & \cdot \\ \cdot & \ldots & \cdot \\ \cdot & \ldots & \cdot \\ -z^{T}[N-1,:] & \cdots & -z^{T}\left[N-n_{a},:\right]\end{array}\right]\right]$

4- Compute the residual as

$$
e_{i v}[k]=A\left(q^{-1}\right) y[k]-B\left(q^{-1}\right) u[k]
$$

where $A\left(q^{-1}\right)$ and $B\left(q^{-1}\right)$ are extracted from $\hat{\theta_{i v}}$

5-Compute a new residual vector $e_{s}[k]$ as:

$$
e_{s}[k]=e_{i v 1}[k] \ldots+e_{i v p}[k]
$$

6- Estimate a SISO AR model of order $p^{*}(n a+n b)$ for $e_{s}[k]:$

$$
e_{S}[k]=F^{-1}\left(q^{-1}\right) e[k]
$$

where

$$
F\left(q^{-1}\right)=1+f_{1} q^{-1}+\ldots . .+f_{p^{*}(n a+n b)^{q}} q^{-p^{*}(n a+n b)}
$$

7- Perform SISO filtering on the components of $u[k]$ and $y[k]$ :

$$
\begin{gathered}
u_{i f}[k]=F^{-1}\left(q^{-1}\right) u_{i}[k] \quad i=1 \text { to } m \\
y_{j f}[k]=F^{-1}\left(q^{-1}\right) y_{j}[k] \quad j=1 \text { to } p
\end{gathered}
$$

8- Compute the auxilary filtred model:

$$
z_{f}[k]=A^{-1}\left(q^{-1}\right) B\left(q^{-1}\right) u_{f}[k]
$$

9- Estimate the final parameters by the IV method using the filtered signals.

$$
\hat{\theta}=\left[\Psi_{f}^{T} \Phi_{f}\right]^{-1} \Psi_{f}^{T} Y_{f}
$$

Where

$$
\begin{aligned}
& \Phi_{f}=\left[\Phi_{y f} \vdots \Phi_{u f}\right] \\
& \Psi_{f}=\left[\Phi_{z f} \vdots \Phi_{u f}\right]
\end{aligned}
$$

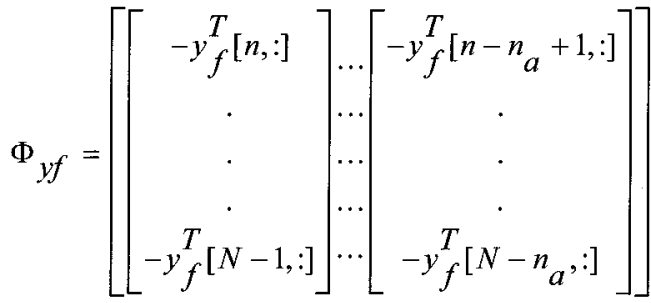

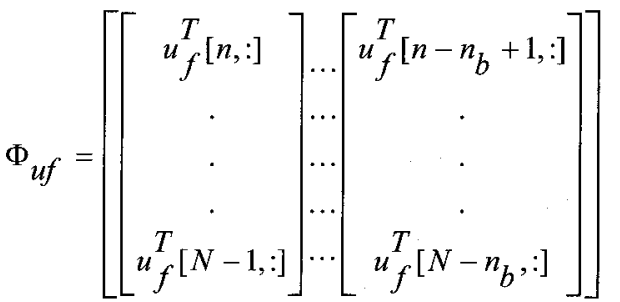

$$
\begin{aligned}
& \left.\Phi_{z f}=\left[\begin{array}{cc|c|c}
-z_{f}^{T}[n,:] & \ldots & -z_{f}^{T}\left[n-n_{a}+1,:\right] \\
\cdot & \ldots & \cdot \\
\cdot & \cdots & \cdot \\
\cdot & \cdots & \cdot \\
-z_{f}^{T}[N-1,:] & \cdots & -z_{f}^{T}\left[N-n_{a},:\right]
\end{array}\right]\right]
\end{aligned}
$$

\section{The Extended SRIV Algorithm}

The SISO SRIV is concerned with the problem of 
estimating the model parameters in terms of the following least squares cost function:

$$
J=\sum_{k=1}^{N} \hat{e}[k]
$$

where $e[k]$ is the following error function obtained directly by inspection of the model

$$
\hat{e}[k]=y[k]-\frac{\hat{B}\left(q^{-1}\right)}{\hat{A}\left(q^{-1}\right)} u[k]
$$

while $\mathrm{N}$ is the total sample size and the "hat" indicates estimated values.

This error function is clearly nonlinear in the parameters of the unknown polynomials. However, it can be written alternatively as:

$$
\hat{e}[k]=\frac{1}{A\left(q^{-1}\right)}\left\{\hat{A}\left(q^{-1}\right) y[k]-B\left(q^{-1}\right) u[k]\right\}
$$

or:

$$
\hat{e}[k]=\hat{A}\left(q^{-1}\right) y^{*}[k]-\hat{B}\left(q^{-1}\right) u^{*}[k]
$$

where $y^{*}[k]$ and $u^{*}[k]$ are the "prefiltered" signals defined as follows,

$$
\begin{aligned}
& \hat{y}[k]=\frac{1}{A\left(q^{-1}\right)} y[k] \\
& \hat{u}[k]=\frac{1}{A\left(q^{-1}\right)} u[k]
\end{aligned}
$$

Equation (18) is now linear-in-the-parameters of the transfer function model, so that normal IV methods could be used to estimate the parameters if it were possible to perform the prefiltering operations in (19) and (20). In practice, of course, the parameters of $A\left(q^{-1}\right)$ are unknown a priori and so this prefiltering operation will be made adaptive, with the algorithm "learning" the parameters of the polynomials in an iterative basis.

The extended SRIV algorithm makes use of the kronicker product and the col $\{$.$\} operator that transforms$ a matrix into a column vector by stacking its columns on top of one another.

Expanding equation (2) gives:

$$
\begin{aligned}
A\left(q^{-1}\right) e[k]= & y[k]+A_{1} y[k-1] \ldots+A_{n a} y[k-n a] \\
& -B_{1} u[k-1] \ldots-B_{n b} u[k-n b]
\end{aligned}
$$

Equation (21) can be written using the kronicker operator as:

$$
\begin{aligned}
A\left(q^{-1}\right) e[k]= & {\left[I_{p} \otimes y[k]^{T}\right] \operatorname{col}\left(I_{p}\right)+\left[I_{p} \otimes y[k-1]^{T}\right] \operatorname{col}\left(A_{1}^{T}\right) \ldots } \\
& +\left[I_{p} \otimes y\left[k-n_{a}\right]^{T}\right] \operatorname{col}\left(A_{n a}^{T}\right)- \\
& {\left[I_{p} \otimes u[k-1]^{T}\right] \operatorname{col}\left(B_{1}^{T}\right) \ldots } \\
& -\left[I_{p} \otimes u\left[k-n_{b}\right]^{T}\right] \operatorname{col}\left(B_{n b}^{T}\right)
\end{aligned}
$$

Solving for $e[k]$ gives:

$$
\begin{aligned}
& e[k]=A^{-1}\left(q^{-1}\right)\left[I_{p} \otimes y[k]^{T}\right] \operatorname{col}\left(I_{p}\right)+\left[A^{-1}\left(q^{-1}\right)\left[I_{p} \otimes y[k-1]^{T}\right] \ldots+\right. \\
& A^{-1}\left(q^{-1}\right)\left[I_{p} \otimes y\left[k-n_{a}\right]^{T}\right]- \\
& A^{-1}\left(q^{-1}\right)\left[I_{p} \otimes u[k-1]^{T}\right] \ldots \\
& -A^{-1}\left(q^{-1}\right)\left[I_{p} \otimes u\left[k-n_{b}\right]^{T}\right]\left[\begin{array}{c}
\operatorname{col}\left(A_{1}^{T}\right) \\
\cdot \\
\cdot \\
\cdot \\
\operatorname{col}\left(A^{T}\right) \\
\operatorname{col}\left(B_{1}^{T}\right) \\
\cdot \\
\cdot \\
\cdot \\
\operatorname{col}\left(B_{n b}^{T}\right)
\end{array}\right]
\end{aligned}
$$

or simply

$$
e[k]=A^{-1}\left(q^{-1}\right)\left[I_{p} \otimes y[k]^{T}\right] \operatorname{col}\left(I_{p}\right)-\varphi_{f}^{T}[k] \theta
$$

The MIMO SRIV Algorithm is as follows:

Algorithm (SRIV algorithm)

$$
\text { 1-Initialize } A\left(q^{-1}\right)=I_{p}
$$

2- Perform MIMO least squares to get an initial estimate of theta

$$
\hat{\theta}=\left[\Phi_{f}^{T} \Phi_{f}\right]^{-1} \Phi_{f}^{T} Y_{f}
$$

3- Compute the auxiliary signal

$$
z[k]=A^{-1}\left(q^{-1}\right) B\left(q^{-1}\right) u[k]
$$

4- Perform MMO filtering on the signals $u[k]$,

$$
\begin{gathered}
y[k] \text { and } z[k] \\
y_{f}[k]=A^{-1}\left(q^{-1}\right)\left[I_{p} \otimes y[k]^{T}\right] \operatorname{col}\left(I_{p}\right) \\
y_{f}[k]=A^{-1}\left(q^{-1}\right)\left[I_{p} \otimes y[k]^{T}\right] \\
u_{f}[k]=A^{-1}\left(q^{-1}\right)\left[I_{p} \otimes u[k]^{T}\right] \\
z_{f}[k]=A^{-1}\left(q^{-1}\right)\left[I_{p} \otimes z[k]^{T}\right]
\end{gathered}
$$

5-Estimate $\theta$ using $I V$ method 


$$
\hat{\theta}=\left[\Psi_{f}^{T} \Phi_{f}\right]^{-1} \Psi_{f}^{T} Y_{f}
$$

where

$$
Y_{f}=\left[\begin{array}{c}
y_{f f}\left[1+p^{*} n a\right] \\
\cdot \\
\cdot \\
y_{f f}\left[p^{*} N\right]
\end{array}\right]
$$

$\Phi_{f}=\left[\Phi_{y f} \vdots \Phi_{u f}\right]$

$\Psi_{f}=\left[\Phi_{z f} \vdots \Phi_{u f}\right]$

and $\Phi_{u f}, \Phi_{y f}$ and $\Phi_{z f}$ are constructed as

follows:

$$
\Phi_{u f}=\left[\left[\begin{array}{cc}
u_{f}\left[1+p^{*}(n a-k),:\right] & \ldots \\
\cdot & \ldots \\
\cdot & \ldots \\
\cdot & \ldots \\
u_{f}\left[p^{*}(N-k),:\right]
\end{array}\right] \quad k=1 \text { to } n b\right.
$$$$
\Phi_{y f}=\left[\begin{array}{cc}
-y_{f}\left[1+p^{*}(n a-k),:\right] & \ldots \\
\cdot & \ldots \\
\cdot & \ldots \\
-y_{f}\left[p^{*}(N-k),:\right] & \cdots \\
\cdots
\end{array}\right]
$$$$
k=1 \text { to } n a
$$$$
\Phi_{z f}=\left[\left[\begin{array}{cc}
-z_{f}\left[1+p^{*}(n a-k),:\right] & ] \\
\cdot & \ldots \\
\cdot & \ldots \\
\cdot & \cdots \\
-z_{f}\left[p^{*}(N-k),:\right] & \cdots \\
\cdots
\end{array}\right]\right.
$$

$$
k=1 \text { to } n a
$$

6-If no convergence, go to step 3 .

\section{Remark 3.1:}

The convergence test used in the last step of the algorithm is the relative error of the parameters in percent defined as:

$$
100\left\|\frac{\hat{\theta}(i+1)-\theta(i)}{\hat{\theta}}\right\|_{2}<\varepsilon
$$

where $\theta_{(i)}$ denotes the estimated parameter vector at iteration i, and $\varepsilon$ is a given tolerance in percent for terminating the iterative search.

Remark 3.2:

A stability check must be performed for both algorithms to force all the roots of the polynomial $\operatorname{det}\left[A\left(q^{-1}\right)\right]=0$ to lie within the unit circle.

\section{Remark 3.3:}

Steiglitz and McBride [5], [6] have suggested an iterative approach to identify a SISO linear system subject to white noise measurement noise. Extension to MIMO systems can be done as follows:

Algorithm (Extended Steiglitz-McBride)

$$
\text { 1-Initialize } A\left(q^{-1}\right)=I_{p}
$$

2- Perform MIMO filtering on the signals $u[k]$ and $y[k]$

$$
\begin{gathered}
y_{f f}[k]=A^{-1}\left(q^{-1}\right)\left[I_{p} \otimes y[k]^{T}\right] \operatorname{col}\left(I_{p}\right) \\
y_{f}[k]=A^{-1}\left(q^{-1}\right)\left[I_{p} \otimes y[k]^{T}\right] \\
u_{f}[k]=A^{-1}\left(q^{-1}\right)\left[I_{p} \otimes u[k]^{T}\right] \\
\text { 3- Compute } \hat{\theta} \text { using LS method }
\end{gathered}
$$

$$
\hat{\theta}=\left[\Phi_{f}^{T} \Phi_{f}\right]^{-1} \Phi_{f}^{T} Y_{f}
$$

Where

$$
\begin{gathered}
Y_{f}=\left[\begin{array}{c}
y_{f f}\left[1+p^{*} n a\right] \\
\cdot \\
\cdot \\
y_{f f}\left[p^{*} N\right]
\end{array}\right] \\
\Phi_{f}=\left[\Phi_{y f} \vdots \Phi_{u f}\right]
\end{gathered}
$$

and $\Phi_{u f}, \Phi_{y f}$ and are constructed as follows:
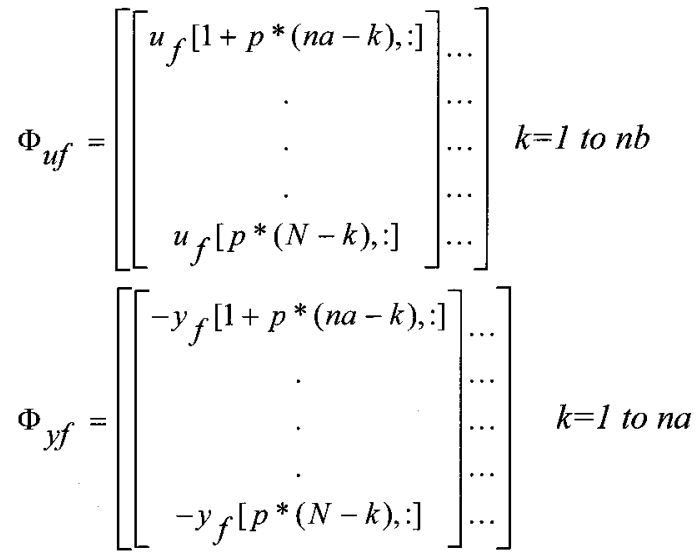

4-If no convergence, go to step 2.

The Steiglitz-McBride technique is therefore close to 
the SRIV technique. The main difference lies in the fact that the SRIV method uses the IV method while the Steiglitz-McBride technique utilizes the LS method only.

\section{Examples}

The features of the MIMO SRIV are illustrated in two examples. The first is a simulation of a linear plant and the second is a simulation of a laboratory nonlinear quadruple tank process.

\subsection{Simulation Example}

A simulation example is presented to illustrate the performance of the MIMO SRIV method as compared to that of the MIMO IV4 and MIMO Least squares estimation methods. Let's consider the 2-input 2-output process (ie, $\mathrm{p}=\mathrm{m}=2$ ) described in LMFD as:

$$
y[k]=A^{-1}\left(q^{-1}\right) B\left(q^{-1}\right) u[k]+e[k]
$$

where

$$
\begin{aligned}
A\left(q^{-1}\right) & =\left[\begin{array}{ll}
1 & 0 \\
0 & 1
\end{array}\right]+\left[\begin{array}{cc}
0.5 & -0.4 \\
0.3 & -0.6
\end{array}\right] q^{-1}+\left[\begin{array}{cc}
-0.1 & -0.3 \\
0.2 & 0.3
\end{array}\right] q^{-2} \\
& =\left[\begin{array}{cc}
1+0.5 q^{-1}-0.1 q^{-2} & -0.4 q^{-1}-0.3 q^{-2} \\
0.3 q^{-1}+0.2 q^{-2} & 1-0.6 q^{-1}+0.3 q^{-2}
\end{array}\right] \\
B\left(q^{-1}\right) & =\left[\begin{array}{cc}
-0.1 & -0.9 \\
0.2 & 0.3
\end{array}\right] q^{-1}+\left[\begin{array}{cc}
-0.8 & -0.3 \\
0.1 & 0.7
\end{array}\right] q^{-2} \\
& =\left[\begin{array}{cc}
-0.1 q^{-1}-0.8 q^{-2} & -0.9 q^{-1}-0.3 q^{-2} \\
0.2 q^{-1}+0.1 q^{-2} & 0.3 q^{-1}+0.7 q^{-2}
\end{array}\right]
\end{aligned}
$$

The aim is to estimate the matrix polynomials $A\left(q^{-1}\right)$ and $B\left(q^{-1}\right)$ from I/O data contaminated by white noise. A PRBS data sequence of length $\mathrm{N}=1000$ is used to excite the system.

A Monte Carlo simulation of 100 experiments has been performed for signal to noise ratio equal to $10 \mathrm{db}$ for both outputs.

The Monte Carlo Simulation (MCS) results are presented in Table 1 where the mean and standard deviation of the estimated parameters are displayed.

\begin{tabular}{|c|c|c|c|c|}
\hline & \multicolumn{2}{|c|}{ MIMO SRIV } & \multicolumn{2}{|c|}{ MIMO IV4 } \\
\hline$\hat{A}_{1}$ & {$\left[\begin{array}{l}0.5029 \pm 0.0235 \\
0.3040 \pm 0.0265\end{array}\right.$} & $\left.\begin{array}{l}-0.3974 \pm 0.0279 \\
-0.6018 \pm 0.0274\end{array}\right]$ & {$\left[\begin{array}{l}0.5018 \pm 0.0256 \\
0.3057 \pm 0.0267\end{array}\right.$} & $\left.\begin{array}{l}-0.3982 \pm 0.0302 \\
-0.5970 \pm 0.0319\end{array}\right]$ \\
\hline$\hat{A}_{2}$ & {$\left[\begin{array}{l}-0.0974 \pm 0.0250 \\
0.1988 \pm 0.0285\end{array}\right.$} & $\left.\begin{array}{c}-0.3024 \pm 0.0292 \\
0.2995 \pm 0.0241\end{array}\right]$ & {$\left[\begin{array}{l}-0.0979 \pm 0.0283 \\
0.2030 \pm 0.0333\end{array}\right.$} & $\left.\begin{array}{c}-0.3007 \pm 0.0312 \\
0.2955 \pm 0.0281\end{array}\right]$ \\
\hline$\hat{B}_{1}$ & {$\left[\begin{array}{c}-0.0979 \pm 0.0147 \\
0.2010 \pm 0.0173\end{array}\right.$} & $\left.\begin{array}{c}-0.9008 \pm 0.0138 \\
0.2999 \pm 0.0158\end{array}\right]$ & {$\left[\begin{array}{l}-0.0974 \pm 0.0169 \\
0.2001 \pm 0.0219\end{array}\right.$} & $\left.\begin{array}{c}-0.9010 \pm 0.0169 \\
0.2979 \pm 0.0195\end{array}\right]$ \\
\hline$\hat{B}_{2}$ & {$\left[\begin{array}{l}-0.8001 \pm 0.0181 \\
0.0959 \pm 0.0215\end{array}\right.$} & $\left.\begin{array}{l}-0.3012 \pm 0.0222 \\
0.6948 \pm 0.0277\end{array}\right]$ & {$\left[\begin{array}{l}-0.8016 \pm 0.0191 \\
0.0969 \pm 0.0232\end{array}\right.$} & $\left.\begin{array}{l}-0.3010 \pm 0.0275 \\
0.6957 \pm 0.0275\end{array}\right]$ \\
\hline
\end{tabular}

It can be seen from Table 1 that both the MIMO SRIV and the IV4 algorithms deliver unbiased and quite accurate results.
Table 1. MCS results

To see the influence of the noise level on parameter estimation, some Monte Carlo simulations of 100 experiments have been performed for different values of SNR ratios varying from 1 to $20 \mathrm{~dB}$. For each run of a Monte Carlo Simulation new noise sequences are generated in order to give independent realizations.

The performance index used for comparison is the Mean Normalized Errors (MNE) which is a measure of bias of the estimates from the true value and is defined as:

$$
M N E=100 \frac{\left\|\theta-\theta_{\text {mean }}\right\|_{2}}{\|\theta\|_{2}}
$$

where $\theta_{\text {mean }}$ is the mean of the estimation parameter value and $\theta$ is the true parameter value.

The results are shown in Table 2.

Table 2. MNE for different values of SNRs.

\begin{tabular}{|c|c|c|c|}
\hline SNR (dB) & $\begin{array}{c}\text { MNE } \\
\text { (SRIV) }\end{array}$ & $\begin{array}{c}\text { MNE } \\
\text { (IV4) }\end{array}$ & MNE (LS) \\
\hline 1 & 1.7760 & 2.7446 & 44.4156 \\
\hline 5 & 0.6392 & 0.8673 & 30.6596 \\
\hline 10 & 0.5536 & 0.6188 & 16.9639 \\
\hline 15 & 0.2582 & 0.3429 & 8.4333 \\
\hline 20 & 0.0750 & 0.0761 & 3.5382 \\
\hline
\end{tabular}


From Table 2 we can see that the MIMO SRIV is more immune to noise than MIMO IV4. Of course the MIMO Least Squares gives bad estimates as expected.

\subsection{Quadruple tank process}

The system under consideration is a laboratory pilot plant [7] composed of four interconnected water tanks and its schematic diagram is shown in figure 1 . Such a process is widely used in industrial applications where we need to maintain a desired level of liquid in a recipient as is the case in chemical or pharmaceutical engineering.

The process inputs are $u_{1}$ and $u_{2}$ (input voltages to the pumps) and the outputs are $h_{1}$ and $h_{2}$ (voltages from level measurement devices). The position of the three-way valves ( $\gamma_{1}$ and $\gamma_{2} \in[0,1]$ ) determine the portion of the output flow going into the upper tank from each pump.

Using the mass balance equations and Bernoulli's law, the system can be described by the following differential equations:

$$
\left\{\begin{array}{l}
\frac{d h_{1}}{d t}=-\frac{a_{1}}{A_{1}} \sqrt{2 g h_{1}}+\frac{a_{3}}{A_{1}} \sqrt{2 g h_{3}}+\frac{\gamma_{1} k_{1}}{A_{1}} v_{1} \\
\frac{d h_{2}}{d t}=-\frac{a_{2}}{A_{2}} \sqrt{2 g h_{2}}+\frac{a_{4}}{A_{2}} \sqrt{2 g h_{4}}+\frac{\gamma_{2} k_{2}}{A_{2}} v_{2} \\
\frac{d h_{3}}{d t}=-\frac{a_{3}}{A_{3}} \sqrt{2 g h_{3}}+\frac{\left(1-\gamma_{2}\right) k_{2}}{A_{3}} v_{2} \\
\frac{d h_{4}}{d t}=-\frac{a_{4}}{A_{4}} \sqrt{2 g h_{4}}+\frac{\left(1-\gamma_{1}\right) k_{1}}{A_{4}} v_{1}
\end{array}\right.
$$

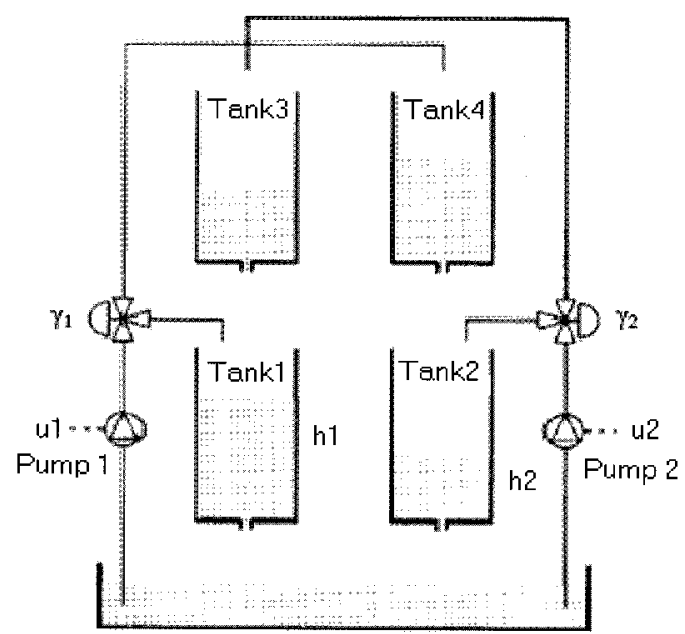

Fig. 1. Schematic diagram of the 4 tank process
The numerical values of the plant parameters [7] are listed in Table3.

Table 3. Model parameters of the quadruple tank

\begin{tabular}{|c|c|c|}
\hline Variable & Symbol & Value \\
\hline \multirow{2}{*}{ Tanks cross section areas } & $\mathrm{A}_{1,}, \mathrm{~A}_{3}$ & $28 \mathrm{~cm}^{2}$ \\
& $\mathrm{~A}_{2,} \mathrm{~A}_{4}$ & $28 \mathrm{~cm}^{2}$ \\
\hline Cross sectional areas of & $\mathrm{a}_{1,} \mathrm{a}_{3}$ & $0.071 \mathrm{~cm}^{2}$ \\
the output holes & $\mathrm{a}_{2,}, \mathrm{a}_{4}$ & $0.057 \mathrm{~cm}^{2}$ \\
\hline \multirow{2}{*}{ Outflow coefficients } & $\mathrm{k}_{1}$ & $3.33 \mathrm{~cm}^{3} /(\mathrm{V} . \mathrm{s})$ \\
& $\mathrm{k}_{2}$ & $3.35 \mathrm{~cm}^{3} /(\mathrm{V} . \mathrm{s})$ \\
\hline Valves setting & $\gamma_{1}$ & 0.7 \\
coefficients & $\gamma_{2}$ & 0.6 \\
\hline Level sensor coefficient & $\mathrm{K}_{\mathrm{c}}$ & $0.5 \mathrm{~V} / \mathrm{cm}$ \\
\hline Gravitation constant & $\mathrm{g}$ & $981 \mathrm{~cm} / \mathrm{s}^{2}$ \\
\hline
\end{tabular}

The quadruple tank process is simulated using simulink as shown in figure 2. A 10 bit analog to digital converter with a $[0,10]$ range is simulated using a zero-order hold block followed by quantizer and saturation blocks. Whereas a 10 bit digital to analog converter is simulated using a quantizer followed by a saturation block.

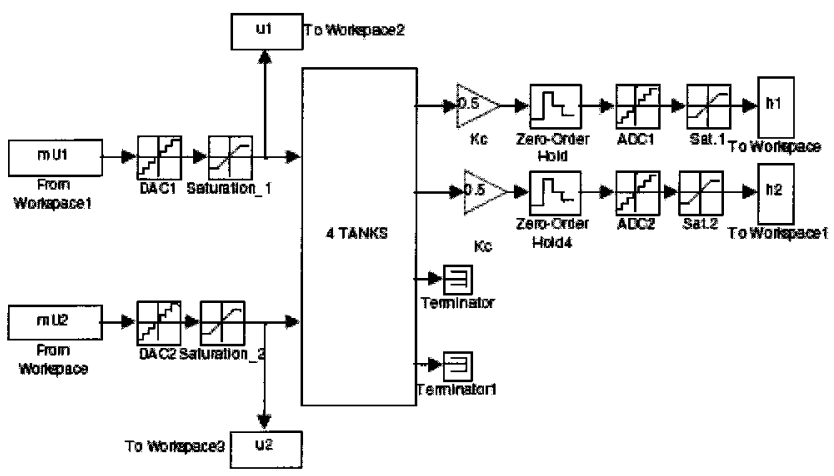

Fig. 2. Data acquisition for the simulated process

To identify a two-input two-output linear model of this nonlinear process around the chosen steady state operating point given in Table 4 we have followed the following steps:

1) the plant is driven to the steady state operating point by applying constant voltages $u_{10}=3$ volts and

$$
u_{20}=3 \text { volts. }
$$

2) then a \pm 0.1 volts PRBS signal is added to each input to excite the system.

3) the I/O data are collected then the steady state values from Table 1 are subtracted from them. 
4) $10 \mathrm{~dB}$ white noise is added to the output data.

5) finally the MIMO SRIV algorithm is applied to the final noisy $\mathrm{I} / \mathrm{O}$ data $\Delta h_{i}$ and $\Delta u_{i}$

where

$$
\Delta h_{i}=h_{i}-h_{i 0}, \Delta u_{i}=u_{i}-u_{i 0} \text { and } i \in[1,2] \text {. }
$$

The obtained input and output data of the experiment are shown in figure3.

Table 4. Steady state values

\begin{tabular}{|l|l|}
\hline Steady state operating point & \multicolumn{1}{|c|}{ Value } \\
\hline $\mathbf{h}_{10}, \mathbf{h}_{\mathbf{2 0}}$ & $12.24,12.77 \mathrm{~cm}$ \\
\hline $\mathbf{h}_{\mathbf{3 0}}, \mathbf{h}_{\mathbf{4 0}}$ & $1.64,1.40 \mathrm{~cm}$ \\
\hline $\mathbf{u}_{10}, \mathbf{u}_{\mathbf{2 0}}$ & $3,3 \mathrm{~V}$ \\
\hline
\end{tabular}
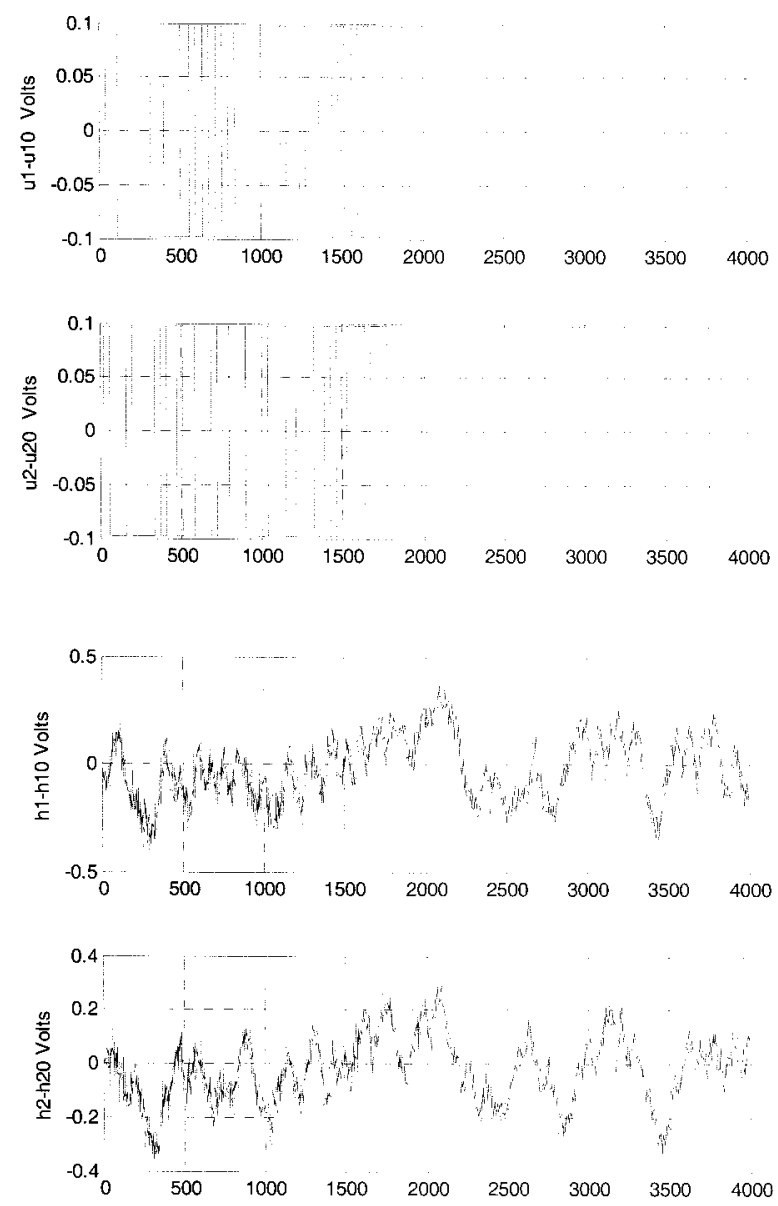

Fig. 3. Noisy Input and Output data

The obtained two-input two-output LMFD model of the quadruple tank process around the chosen operating point using the MIMO SRIV is:

$$
\Delta h[k]=A^{-1}\left(q^{-1}\right) B\left(q^{-1}\right) \Delta u[k]
$$

$$
\begin{aligned}
& A\left(q^{-1}\right)=\left[\begin{array}{cc}
1-0.9778 q^{-1} & -0.0096 q^{-1} \\
-0.0083 q^{-1} & 1-0.9831 q^{-1}
\end{array}\right] \\
& B\left(q^{-1}\right)=\left[\begin{array}{ll}
0.0450 q^{-1} & 0.0115 q^{-1} \\
0.0022 q^{-1} & 0.0339 q^{-1}
\end{array}\right]
\end{aligned}
$$

The computed mean square error [2] using MIMO SRIV is 0.00181559157552 whereas MIMO IV4 and MIMO ARX gave us 0.00190628448072 and 0.01134863885998 respectively.

\section{Conclusion}

This paper has presented an extension of the SRIV algorithm to MIMO systems described by a Left Matrix Fraction Description using the Kronicker product. Block filtering of the input/output as well as iterativity are the main features of the algorithm. The Monte Carlo simulations that are conducted for different signal to noise ratios revealed the superiority of the algorithm over the MIMO IV4 and the MIMO least squares algorithms.

\section{References}

[1] L. Ljung, System Identification: Theory for the user, Prentice Hall, 1999.

[2] L. Ljung, System identification toolbox for Matlab, Version 6.1.2, www.mathworks.com, 2005.

[3] P. Young, and A.J. Jakeman, Refined instrumental variable methods of time-series analysis, International Journal of Control 29, 621-644, 1979.

[4] P. Young, An instrumental variable approach to ARMA model identification and estimation, Proceedings of the 14th IFAC Symposium on System identification (SYSID'2006 Newcastle, Australia), 2006.

[5] K. Steiglitz and. L.E. McBride, A technique for the identification of linear systems, IEEE Transactions on Automatic Control, 10, 461-464, 1965.

[6] P. Stoica, and T. Soderstrom, 1981, The Steiglitz McBride identification algorithm revisited, IEEE Transactions on Automatic Control, 29, 712-719.

[7] K.H. Johansson, The Quadruple-Tank Process: A multivariable laboratory process with an adjustable zero, IEEE Transactions on Control Systems Technology, Vol.8, No.3, May 2000.

[8] S.D. Fassois, MIMO LMS-ARMAX identification of vibrating structures, Mechanical Systems and Signal Processing,, 15, 723-735, 2001.

[9] A. Nehorai, and M. Morf, Recursive identification Algorithms for Right Matrix Fraction Description 
models, IEEE Transactions on Automatic Control, 29, 1103-1106, 1984.

[10] T. Kailath, Linear Systems, Prentice Hall, 1980.

[11] S.Kim, J.Jin, Y.Park, Approximate ML Detection with Best channel Matrix Selection for MIMO systems, Journal of Electrical Engineering \& Technology, Vol.3, No.2, June 2008.

[12] H. Zabot and K.Hariche, On solvents-based model reduction of MIMO systems, International journal of systems science, 28, 499-505, 1997.

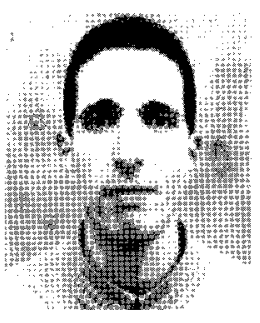

\section{Mohamed Akroum}

He received his Electrical Engineering Degree in 1995 from M'hamed Bougara University (UMBB, Algeria) and his Magister Degree in 1998 from USTHB university (Algeria). He worked two years in the Advanced Technologies Development Center in Algiers, and he is currently a lecturer at UMBB university. His current research interests include MIMO system identification and multivariable automatic control.

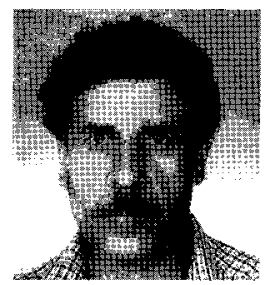

\section{Kamel Hariche}

$\mathrm{He}$ received his M.Sc. and $\mathrm{PhD}$ degrees from the University of Houston in 1978 and 1987 respectively. $\mathrm{He}$ is currently a Professor at UMBB University. His research is related to linear and nonlinear systems as well as Automatic control of MIMO systems. 